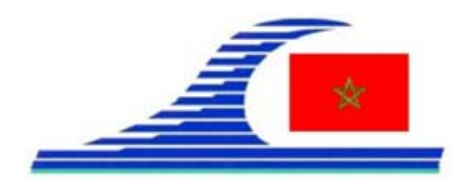

Conférence Méditerranéenne Côtière et Maritime

EDITION 2, TANGER, MAROC (2011)

Coastal and Maritime Mediterranean Conference

Disponible en ligne - http://www.paralia.fr - Available online

\title{
Clapage de sédiments non-cohésifs sous courant : simulations versus expérimentations
}

\section{Duc Hau NGUYEN ${ }^{1,2}$, Sylvain GUILLOU ${ }^{1}$, Kim Dan NGUYEN ${ }^{2}$, Damien PHAM VAN BANG ${ }^{2}$}

\section{Université de Caen, LUSAC (EA4253),}

Site Universitaire de Cherbourg, BP 78, 50130 Octeville, France.

sylvain.guillou@unicaen.fr; duc-hau.nguyen@unicaen.fr

2. Université Paris-Est, LSHV, (Joint Research Unit EDF R\&D-CETMEF-ENPC),

6 quai Watier, BP 49, Bat. K, F-78401 Chatou, France.

\section{Mots-clés :}

Clapage - Transport sédimentaire - Modélisation numérique - Modèle à deux phases

\section{Introduction}

La maintenance des chenaux de navigation et des zones portuaires implique la réalisation d'opérations de dragage. Les produits de dragages sont souvent déposés en mer (opération de clapage) pouvant induire des nuisances sur l'environnement. Le phénomène de clapage comporte principalement trois phases (BOUTIN, 2000) : la phase de chute (soumise aux courants); l'impact sur le fond et la génération d'un courant de densité ; la propagation des courants de densité et la décantation des sédiments. Nous simulons ce phénomène à l'aide d'un modèle à deux phases adapté aux milieux denses en différentiant les deux constituants. Les études sur le clapage avec ce modèle ont démarré dans un cas sans courant (GUILLOU et al., 2011). Les travaux présentés ici portent sur une étude systématique de la dynamique de chute et de transport en fonction du courant relative à la configuration expérimentale utilisée par VILLARET et al. (1998).

\section{Modèle à deux phases}

L'utilisation de l'approche à deux phases pour traiter la problématique hydrosédimentaire est apparue dans les années 90 (NGUYEN et al., 2009, et références incluses). Dans cette approche le mélange eau/sédiment est défini comme constitué de deux phases : une phase continue, l'eau, et une phase dispersée, les sédiments. Dans l'approche à deux phases Eulérienne-Eulérienne (fluide - particule solide), on considère que les mouvements de chaque phase sont régis respectivement par des équations de conservation de la masse et de la quantité de mouvements. Des termes de transferts sont introduits pour modéliser l'échange entre les phases. Ainsi est conçu notre modèle (BARBRY et al., 2000 ; NGUYEN et al., 2009) dit à "deux fluides" qui est adapté aux milieux denses en différentiant les deux constituants et inclut les effets de la turbulence 
La connaissance de la Mer :

un vecteur du développement durable en Méditerranée

(CHAUCHAT et GUILLOU, 2008). Le lecteur trouvera une description des équations du modèle dans les références ci-avant.

\section{Configuration de l'étude}

Le montage expérimental de VILLARET et al. (1998) est constitué d'un canal (canal n ${ }^{\circ}$ 5 du LNHE-EDF : $80 \mathrm{~m} \times 1.5 \mathrm{~m} \times 1.5 \mathrm{~m}$ ) équipé d'un dispositif d'injection de sédiment placé à $15 \mathrm{~cm}$ sous la surface libre et piloté par un PC (figure 1a). A $t=0$, un volume initial de mélange eau-sédiment à une concentration désirée est lâché dans l'eau. Une caméra synchronisée prend des clichés du panache turbide généré (figure 1b), pendant qu'un système enregistre la concentration en plusieurs points du canal disposés dans le canal (figure 1a). Le tableau 1 donne les différentes conditions expérimentales.
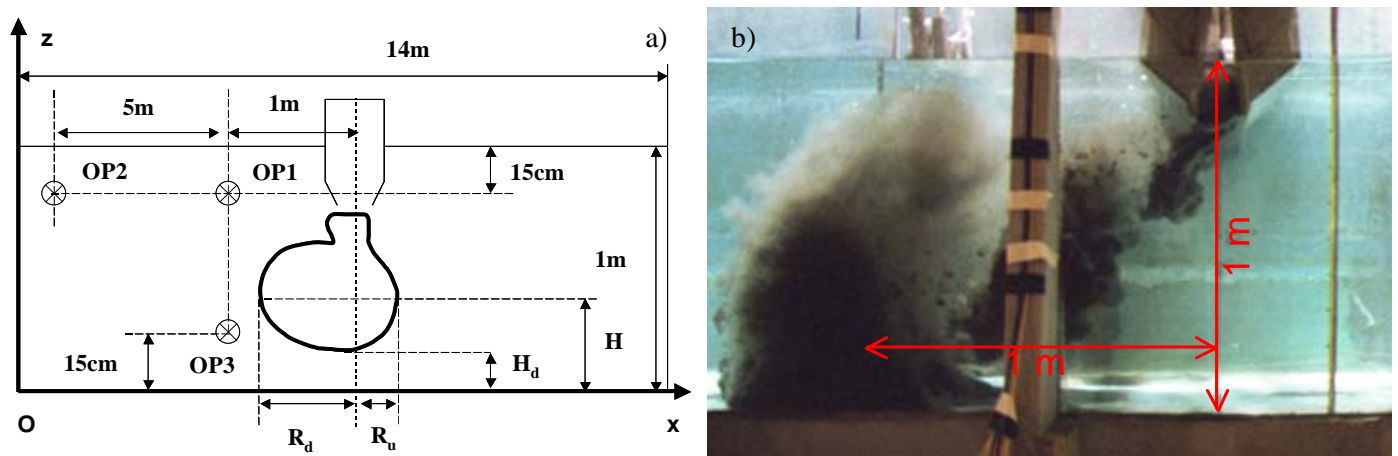

Figure 1. Configuration expérimentale :(a) localisation des capteurs optiques de turbidité (OP) ; (b) mud dumping (VILLARET et al., 1998).

Tableau 1. Caractéristiques des essais 100\% sable avec courant ambiant : Winj est la vitesse d'injection à la sortie du réservoir, $D_{p}$ est le diamètre des particules (90 $\mu \mathrm{m}$ ou $160 \mu \mathrm{m}), \rho_{\mathrm{s}}$ est la masse volumique de la phase solide $\left(2650 \mathrm{~kg} / \mathrm{m}^{3}\right), \mathrm{Cm}$ est la concentration du mélange dans le réservoir, $\mathrm{Vr}$ est le volume de matériel relâché, Uc est la vitesse ambiante (VILLARET et al., 1998).

\begin{tabular}{llllllllllll}
\hline Tests & $\boldsymbol{e 6}$ & $\boldsymbol{e 1 1}$ & $\boldsymbol{e 1 2}$ & $\boldsymbol{e 1 3}$ & $\boldsymbol{e 1 4}$ & $\boldsymbol{e 1 5}$ & $\boldsymbol{e 1 6}$ & $\boldsymbol{e 1 7}$ & $\boldsymbol{e 1 8}$ & $\boldsymbol{e 1 9}$ & $\boldsymbol{e 2 0}$ \\
\hline $\boldsymbol{W}_{\text {inj }}(\mathbf{m} / \mathbf{s})$ & 0.6 & 0.79 & 0.89 & 0.79 & 0.89 & 0.79 & 0.89 & 0.79 & 0.89 & 0.79 & 0.89 \\
$\boldsymbol{D}_{\boldsymbol{p}}(\boldsymbol{\mu m})$ & 90 & 90 & 160 & 90 & 160 & 90 & 160 & 160 & 90 & 160 & 90 \\
$\mathbf{C m}(\mathbf{g} / \mathbf{l})$ & 350 & 450 & 450 & 450 & 450 & 450 & 450 & 450 & 450 & 450 & 450 \\
$\boldsymbol{V r}(\boldsymbol{l})$ & 45 & 60 & 60 & 60 & 60 & 60 & 60 & 60 & 60 & 60 & 60 \\
$\boldsymbol{U c}(\mathbf{c m} / \mathbf{s})$ & 0 & 0 & 0 & 10 & 10 & 20 & 20 & 15 & 15 & 25 & 25 \\
\hline
\end{tabular}

\section{Résultats}

Le domaine d'étude couvre une zone de 14 mètres horizontalement centrée sur le rejet et de 1 mètre en hauteur. Un maillage régulier de $61 \times 1401$ nœuds ainsi qu'un pas de temps de $\Delta t=0,001 \mathrm{~s}$ sont utilisés. Les paramètres sont ceux du tableau 1. Un schéma UPWIND premier ordre est utilisé et les frontières latérales sont considérées ouvertes. A la position d'injection (diamètre d'injection de $10 \mathrm{~cm}$ ), le flux de mélange est imposé en considérant une vitesse avec un profil de Poiseuille de vitesse maximale $W_{i n j}$ donnée dans le tableau 1 (GUILLOU et al., 2011). 


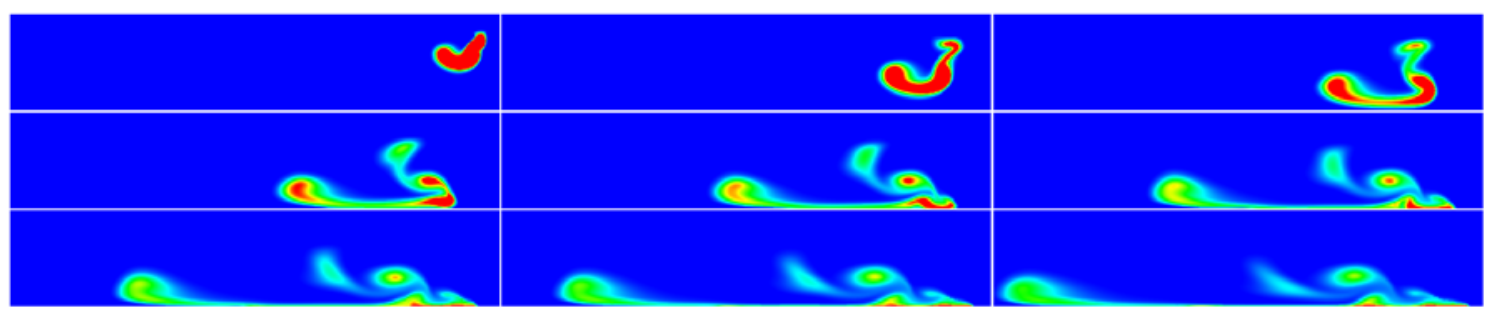

C(gil)

010203040506070

Figure 2. Isocontours de concentration : Résultats de simulation pour l'essai e20 à différents instants.
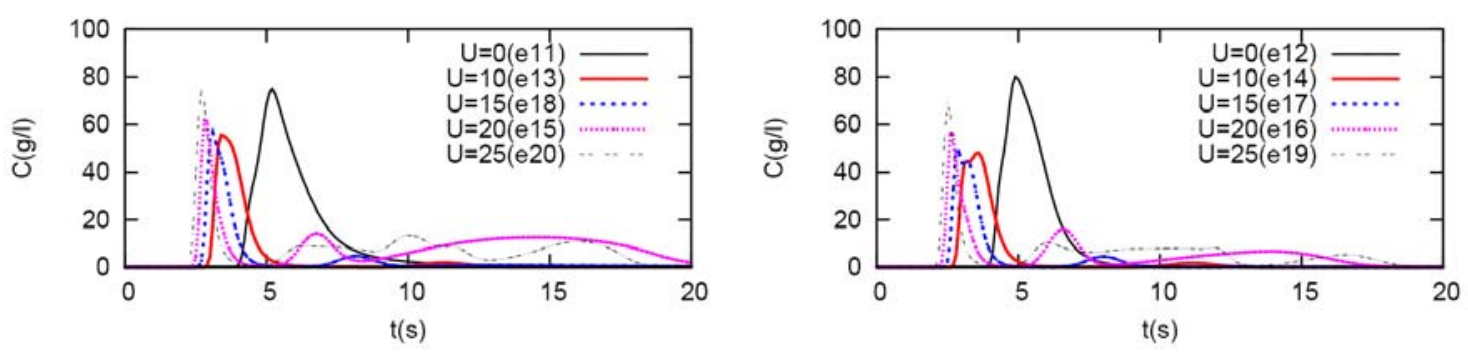

Figure 3. Evolution temporelle de la concentration au fond (sonde OP3) pour le sable 1 (à gauche) et le sable 2 (à droite).

Tableau 2. Temps de chute (Tc) et diamètre de rejet (Dr) en fin de chute, et instant du pic de concentration (Tpic) et concentration (Cpic) au point de sondage OP3 : résultats des simulations (num) et valeurs expérimentales (VILLARET et al., 1998).

\begin{tabular}{|c|c|c|c|c|c|c|c|c|c|c|}
\hline $\begin{array}{l}\text { Tests } \\
\text { Sable }\end{array}$ & e11 & e13 & $\begin{array}{r}\boldsymbol{e 1 8} \\
1\end{array}$ & e15 & $e 20$ & $e 12$ & e14 & $\begin{array}{r}e 17 \\
2\end{array}$ & e16 & e19 \\
\hline Uc $(\mathrm{cm} / \mathrm{s})$ & 0 & 10 & 15 & 20 & 25 & 0 & 10 & 15 & 20 & 25 \\
\hline$T c_{\text {num }}(s)$ & 1.5 & 2.1 & 2.4 & 2.6 & 2.8 & 1.3 & 1.9 & 2.1 & 2.3 & 2.4 \\
\hline$T c_{\text {exp }}(s)$ & 0.95 & 1.54 & 1.50 & 1.6 & 2.2 & 0.72 & 1.95 & 2.62 & 2.10 & 2.20 \\
\hline$D r_{n u m}(m)$ & 0.68 & 0.92 & 1.08 & 1.15 & 1.18 & 0.54 & 0.90 & 1.00 & 1.07 & 1.05 \\
\hline$D r_{e x p}(m)$ & 0.64 & 0.50 & 0.62 & 0.60 & 0.46 & 0.46 & 0.44 & 0.52 & 0.58 & 0.68 \\
\hline $\operatorname{Tpic}_{\text {num }}(\mathrm{s})$ & 5.2 & 3.4 & 2.8 & 3.1 & 2.7 & 4.9 & 3.6 & 2.6 & 2.9 & 2.5 \\
\hline $\operatorname{Tpic}_{\text {exp }}(\mathrm{s})$ & 7.4 & 4.6 & 3.7 & 4.3 & 4.0 & 6.3 & 5.4 & 4.6 & 5.0 & 4.0 \\
\hline Cpic $_{\text {num }}(g / l)$ & 74.8 & 55.5 & 61.7 & 56.9 & 74.1 & 80.1 & 48.1 & 56.4 & 48.7 & 68.7 \\
\hline $\operatorname{Cpic}_{\exp }(g / l)$ & 56.5 & 50 & 89 & 78 & 75 & 48 & 73 & 61 & 84 & 59 \\
\hline
\end{tabular}

La figure 2 présente les isocontours de concentration simulés pour une vitesse de courant Uc de $25 \mathrm{~cm} / \mathrm{s}$ (e20). Les sédiments ne s'étalent presque pas vers l'amont.

Le temps de chute ainsi que le diamètre de rejet au moment de l'impact sont influencés par l'intensité du courant et par la granulométrie (tableau 2). Les temps de chute simulés quoique de l'ordre des temps mesurés, présentent des différences notamment dans le cas sans courant. La méthode d'estimation du temps de chute par prise de photographies, est dépendante de l'intervalle de temps entre deux prises. Celui-ci est de 0,5 s (incertitude de mesure), qui est de l'ordre de grandeur du temps de chute sans courant. L'incertitude sur le diamètre de rejet est quant à elle de $5 \mathrm{~cm}$. La précision de la mesure du temps de 
La connaissance de la Mer :

un vecteur du développement durable en Méditerranée

chute est donc perfectible. D’autre part, le temps de chute simulé est conditionné par la condition d'injection. Par la suite nous raisonnons sur les tendances entre les cas. Plus les particules sont grandes (sable 2) et plus le temps de chute est court et moins le diamètre à l'impact est grand. La progression du temps de chute calculé pour le sable 1 est comparable à celle de l'expérience. Le maximum observé pour le sable 2 sur l'expérience (e17) n'est pas observé sur la simulation. Contrairement à l'expérience, le modèle prédit un temps de chute (pour tous les cas) plus court pour le sable le plus lourd. Au point de sondage OP3, le niveau de turbidité passe par un pic important (témoignant du passage d'un nuage de particules très concentré) puis par plusieurs maxima de moindres importances lors qu'il y a un courant ambiant (figure 3). Ces derniers correspondent au rabattage des nuages de particules initialement à l'amont. La simulation et l'expérience prédisent une différence forte de Tpic (instant du premier pic de concentration) entre le cas sans courant et les cas avec courant (tableau 2). Pour ces derniers cas, Les valeurs de ce paramètre sont assez semblables pour un même type de sable. L'écart du premier pic est plus important lorsque le sable est plus fin. Lorsque le courant augmente, on constate que l'influence de la granulométrie a tendance à s'atténuer. Ceci est également observé sur l'expérience. Le modèle anticipe cependant systématiquement le passage du pic d'au moins $1 \mathrm{~s}$. L'ordre de grandeur de la concentration maximale est cohérent avec l'expérience (de 48 g/l à 89 g/l pour l'expérience, et de 48 g/l à 80 g/l pour la simulation).

Remerciements : Les auteurs remercient le gouvernement vietnamien pour le financement de la thèse de Duc Hau NGUYEN.

\section{Références bibliographiques}

BARBRY N., GUILLOU S., NGUYEN K.D. (2000). Une approche diphasique pour le calcul du transport sédimentaire en milieux estuariens. C.R. Acad. Sci., IIb, 328, pp 793-799.

BOUTIN R. (2000). Dragage et rejets en mer. Les produits de type vase. Presses de l'ENPC. 2000, 307 p.

CHAUCHAT J., GUILLOU S. (2008). On turbulence closures for two-phase sedimentladen flow models. J. Geophys. Res., 113, C11017. doi:10.1029/2007JC004708

GUILLOU S., CHAUCHAT J., PHAM VAN BANG D., NGUYEN D.H., NGUYEN K.D. (2011). Simulation of the dredged sediment's release with a two-phase flow model. Bulletin of the PIANC, 142, 25-33, ISSN 0374-1001.

NGUYEN K.D., GUILLOU S., CHAUCHAT J., BARBRY N. (2009). A two-phase numerical model for suspended-sediment transport in estuaries. Advances in Water Resources, 32, pp 1187-1196. doi:10.1016/j.advwatres.2009.04.001

VILLARET C., CLAUDE B., DU RIVAU J.D. (1998). Etude expérimentale de la dispersion des rejets par clapage. He-42/98/065/a, LNHE, EDF. 